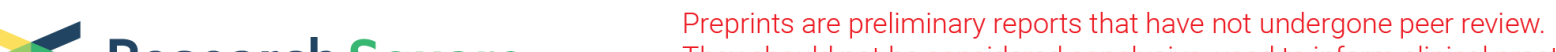

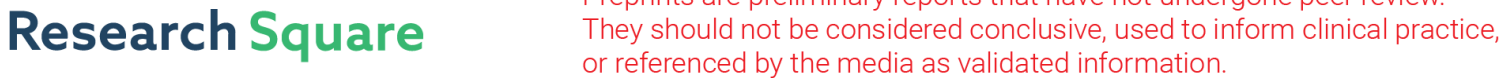

\section{Resurgence risk for malaria, characterization of a recent outbreak in a Amazonian border area -French Guiana/Brazil-}

\section{Emilie MOSNIER ( $\nabla$ emilie.mosnier@gmail.com )}

Centre Hospitalier Andree Rosemon and Aix Marseille University, INSERM, IRD, SESSTIM, Science Economiques \& Sociales de la Santé \& Traitement de l'Information Médicale https://orcid.org/00000002-6004-3323

\section{Isabelle Dusfour}

Unité Contrôle et Adaptation des Vecteurs, Institut Pasteur de la Guyane, Cayenne; INRS-Centre ArmandFrappier santé Biotechnologie, Laval, Canada

\section{Guillaume Lacour}

Unité Contrôle et Adaptation des Vecteurs, Institut Pasteur de la Guyane, Cayenne

\section{Raphael Saldanha}

LIS, ICICT, Fiocruz, Rio de Janeiro

\section{Amandine Guidez}

Unité Contrôl et Adaptation des Vecteurs, Institut Pasteur de la Guyane, Cayenne

\section{Margarete S. Gomes}

Superintendência de Vigilância em Saúde do Amapá, Macapá

\section{Alice Sanna}

Agence Régionale de la Santé de Guyane, Cayenne

\section{Yanouk Epelboin}

Unité Contrôle et Adaptation des Vecteurs, Institut Pasteur de la Guyane, Cayenne

\section{Johana Restrepo}

Service d'Enthomologie de la Direction Démoustication et Action Sanitaires, Collectivité Térritoriale de la Guyane, Cayenne

\section{Damien Davy}

Laboratoire Ecologie, Evolution, Interactions des Systèmes Amazoniens, CNRS, Université de la Guyane, IFREMER, Cayenne

\section{Magalie Demar}

Laboratoire de Parasitologie et Mycologie, Centre Hospitalier Andrée Rosemon, Cayenne

\section{Félix Djossou}

Unité des Maladies Infectieuses et Tropicales, Centre Hospitalier Andrée Rosemon, Cayenne

\section{Maylis Douine}


Centre d'Investigation Clinique Antille Guyane-Inserm 1424, Centre Hospitalier Andrée Rosemon, Cayenne

\section{Vanessa Ardillon}

CIRE Guyane, Cayenne

\section{Mathieu Nacher}

Centre d'Investigation Clinique Antille Guyane -Inserm 1424, Centre Hospitalier Andrée Rosemon, Cayenne

\section{Lise Musset}

Laboratoire de parasitologie, Centre National de Référence du Paludisme, Pôle Zones Endémiques, WHO Collaborating Center for Surveillance of Antimalarial Drug Resistance, Institut Pasteur de la Guyane, Cayenne

\section{Emmanuel Roux}

ESPACE-DEV, IRD, Université de Montpellier, de la Réunion, de la Guyane, des Antilles, LIS, ICICT, Fiocruz, Rio de Janeiro

\section{Research article}

Keywords: Plasmodium vivax, Anopheles darlingi, French Guiana, Brazil, border, outbreak investigation, South American Indians, Malaria, Amazonia

Posted Date: March 9th, 2020

DOI: https://doi.org/10.21203/rs.3.rs-16296/v1

License: (9) This work is licensed under a Creative Commons Attribution 4.0 International License. Read Full License

Version of Record: A version of this preprint was published at BMC Infectious Diseases on May 26th, 2020. See the published version at https://doi.org/10.1186/s12879-020-05086-4. 


\section{Abstract}

Background In 2017, inhabitants along the border between French Guiana and Brazil were affected by a malaria outbreak primarily due to Plasmodium vivax ( Pv ). While malaria cases have steadily declined between 2005-2016 in this Amazonian region, a resurgence was observed in 2017.

Methods Two investigations were performed according to two different spatial scales and type of information details. Firstly, a local study on the French Guiana border, which enabled a thorough investigation of the malaria cases treated at the local village health center and of the entomological situation in the most affected neighborhood and secondly a regional and cross-border study, which enabled exploration of the regional spatial-temporal epidemic dynamic. Number and location of malaria cases were estimated using French and Brazilian surveillance systems.

Results On the French Guiana side of the border in Saint Georges de l'Oyapock, the attack rate was 5.5\% $(n=219 / 4000)$, reaching 51.4\% $(n=90 / 175)$ in one Amerindian neighborhood. Entomological findings suggest a peak of Anopheles darlingi density in August and September. Two female An. darlingi $(n=2 / 1104,0.18 \%)$ were found Pv -positive during this peak. During the same period, aggregated data from passive surveillance conducted by Brazilian and French Guianese border health centers identified 1,566 cases of $\mathrm{Pv}$ infection. Temporal distribution during the 2007-2018 period displayed seasonal patterns with a peak in November 2017. Four clusters were identified among the epidemic profiles of the localities of the cross-border area. All localities of the first two clusters were Brazilian. The localization of the first cluster suggests an onset of the outbreak in the Amerindian reservation, subsequently expanding to French Amerindian neighborhoods and to non-Native communities.

Conclusions The current findings demonstrate a potential increase in malaria cases in an area with otherwise declining numbers. This is a transborder area where human mobility and remote populations challenge malaria control programs. This investigation illustrates the importance of international border surveillance and collaboration for malaria control, particularly in Amerindian villages and mobile populations.

\section{Background}

After two decades of global decreases in malaria incidence, rates are increasing for the first time since 2016 [1]. In the Americas, the largest increase was recorded in Brazil and Venezuela [1]. Thus, malaria remains a public health challenge in South America. In Brazil, transmission is mainly entrenched in the Amazon Basin, which accounts for $99.5 \%$ of Brazil's malaria burden [2].

French Guiana is a European overseas, malaria-endemic territory. The Oyapock river forms the border between French Guiana and Brazil. This area has engaged in a regional malaria control program [3], which faces challenges in controlling malaria among autochthonous and illegal gold miner populations living generally in remote areas and along the borders with Surinam and Brazil [2-5]. Along the Oyapock river, Plasmodium falciparum has declined over the past several years and P. vivax is now responsible for 
most malaria cases [6, 7]. Although transmission decline is observed on a global scale, local heterogeneities persist and must be addressed through targeted control initiatives in order to achieve malaria elimination $[8,9]$. This is particularly important in border areas, which complicate effective implementation of malaria control interventions $[2,10]$. Anopheles darlingi is the predominant species and a major malaria vector in this territory $[11,12]$.

The aim of this article is to describe the malaria outbreak in 2017 through an entomo-epidemiological investigation in the Saint Georges de l'Oyapock (STG) area along the Oyapock river. The investigation is associated with a retrospective study using a spatio-temporal analysis of malaria cases data from surveillance health systems of French Guiana and Brazil.

\section{Outbreak detection}

The malaria surveillance system in French Guiana is based on three data sources: remote health centers (CDPS) reports and laboratory and military health services notification. Since the end of May 2017, data from the CDPS of Saint Georges de l'Oyapock showed a slow and persistent increase of P. vivax cases in the Native neighborhood of Trois Palétuviers. These clusters and the subsequent local outbreak have no apparent link to any other transmission sites in French Guiana, nor with the epidemiological situation in the municipality of Oiapoque, an immediate neighbor on the Brazilian side of the border. The transmission continued, and between September and October 2017, increases in the number of malaria cases were observed in downtown STG, French Guiana. This number largely exceeded the expected cases over the past three years for this endemic area [7].

Retrospectively, the data provided by the Brazilian malaria epidemiological surveillance information system (SIVEP-Malária) revealed an increase in the number of cases of malaria infections in the Oiapoque municipality, including among the Uaçá, Galibi and Juminã indigenous territories (Amapá, Brazil).

\section{Methods}

\section{Study area and population}

On the French side, the cross-border Oyapock region includes three municipalities: Camopi, STG and Ouanary, with approximately 1,700, 4,000 and 100 residents respectively in 2017 according to the STG health center census. The region marks the north-eastern border between French Guiana and Brazil (Figure 1). On the Brazilian side, the cross-border area includes the municipality of Oiapoque, which holds 25,514 inhabitants according to the 2015 estimation by the Brazilian National Institute of Statistics, IBGE), and lies along the Oyapock river (Figure 1). To the north and east of the Oiapoque municipality, there are a large number of small villages. Amerindian territories (Uaçá, Galibi, Juminã) are predominant. This cross-border area consists of a vast remote territory covered by the Amazonian rainforest associated with highly variable population densities. There is also a great diversity of populations, including Amerindians (mainly Wayãpi, Teko, Palikur, Karipuna, Galibi-Marworno and Galibi tribes), Creoles, 
Saramaka and migrants from other Brazilian states who migrate to the area to work in the gold mining sector (mainly illegal gold mining or border supply zones). Therefore, daily transborder exchanges exist among these populations who share a unique living, fishing and hunting area. The climate is equatorial, with four alternating seasons: a long rainy season from April to June, a dry season from July to December, a short, rainy season from January to February and a short dry season in March. The cities of STG and Oiapoque are persistent, low malaria endemicity areas [7].

\section{Malaria case definition}

Malaria diagnosis was performed in health centers using the Rapid Diagnosis Test (RDT) - SD Bioline ${ }^{\circledR}$ Malaria Ag Pf/Pan in French Guiana, and thick and thin smears or RDTs were used in Brazil. A malaria case was defined as a patient with RDT or microscopy-positive results. Data included passive monitoring of cases in border health centers in French Guiana and Brazil, but also active case detection around positive cases in the city of Oiapoque.

Before treatment with primaquine, a glucose-6-phosphate dehydrogenase (G6PD) deficiency test was conducted at least two weeks after the malaria infection in French Guiana [13].

In French Guiana, P. vivax relapse was defined as having a medical history of malaria within a period of 7 to 90 days since the last malaria diagnosis. This interval was considered adequate in length to distinguish follow-up (0-7 days), relapse (7-90 days) and new infection (>90 days) $[7,14]$.

In Brazil, the absence of a unique patient identification code did not permit the use of the same relapse identification method. However, malaria attacks related to patient follow-up, treatment failures and potential relapses were identified during the medical consultation and denoted as treatment verification slide (Lâmina de Verificação de Cura, LVC) in the database. A malaria attack was considered as an LVC for $P$. vivax if the patient was positive for $P$. vivax and received a treatment against $P$. vivax malaria during the last 60 days. It is worth noting that $P$. vivax relapses are expected to be less likely to occur in Brazil due to a systematic primaquine administration (except for specific cases such as pregnancy), which did not exist on the French Guiana side.

As listed below, two investigations were performed according to two different spatial scales and type of information details:

1. a local study on the French Guiana border, which enabled a thorough investigation of the malaria cases treated at the STG health center and of the entomological situation in the most affected neighborhood;

2. a regional and cross-border study, which enabled exploration of the regional spatial-temporal epidemic dynamic.

\section{Local investigations on the French Guiana side}


Data from medical records of the STG health center allowed a retrospective analysis of epidemics. Malaria cases diagnosed between January 1, 2017 and January 31, 2018 were included, and the following variables analyzed: age, gender, outcome and location of acute $P$. vivax malaria cases and relapses treated in the only STG health center of the study area. Census data were retrieved from the health center in order to calculate the incidence and the attack ratio by neighborhood. Factors associated with risk of attack/relapse were identified by univariate analysis.

\section{Entomological investigations}

The entomological investigation focused on Trois-Palétuviers, a neighborhood of STG experiencing large numbers of malaria cases during this period. Mosquitoes were collected monthly from August to November 2017 over 2 to 3 consecutive nights per month, then identified morphologically. Two octenolbaited Mosquito Magnet ${ }^{\circledR}$ traps were used to collect anopheline species and were supplemented with BG-Sentinel and CDC light traps [15]. The collections were performed periodically from 18:00 to 07:00 am. Intra-domiciliary aspirations were done inside four houses between 19:00 and 20:00 in August.

The infectious status of the Anopheles spp. captured from August to October was investigated. The head and thorax of 1,218 females were dissected and placed in an agitator with grinding beads. The DNA of each sample was extracted using a Magjet Genomic DNA kit (Thermo Scientific, K2722), then 10 female DNA samples were pooled for polymerase chain reaction (PCR) detection. The presence of $P$. falciparum, $P$. vivax and $P$. malariae parasites was investigated by nested PCR using the Snounou et al. method [16]. Individual confirmation was performed for each positive pool.

\section{Regional analysis of the cross-border epidemic dynamic}

Registries

French Guiana data came from border area health centers (Delocalized Centers for Prevention and Care CDPS) in French Guiana.

Brazilian data came from the Brazilian information system dedicated to epidemiological surveillance (Sistema de Informações de Vigilância Epidemiológica da Malária, SIVEP-Malária).

The harmonization of the epidemiological information provided by the French and Brazilian malaria surveillance systems was performed by the cross-border malaria information system described in Saldanha et al., submitted.

\section{Epidemic profile clustering}

In order to describe the spatial-temporal epidemic dynamics in the study area, the epidemic profiles of the cross-border area localities were defined and clustered, and the resulting clusters were represented and interpreted both temporally and spatially. To achieve this, first we selected localities presenting a significant number of cases, followed by each country individually. The method included: 
1. ranking all localities according to their total number of cases during the study period;

2. selecting all localities that contributed up to $90 \%$ of the total number of cases. Time-series of malaria cases were obtained by aggregating the daily case counts on a weekly basis. Locality epidemic profiles were defined by the normalized cumulated numbers of malaria cases. This method highlighted the curves dynamics and facilitated their interpretation. Next, Ward's hierarchical clustering method using Euclidean distance was applied to the epidemic profiles. The results were represented and interpreted in both space and time dimensions.

\section{Ethical approval}

The French Guianese database was anonymized and declared to the Commission Nationale Informatique et Libertés (CNIL) (authorization $N^{\circ} 1939018$ ). Brazil's surveillance registries database was anonymized prior to being sent. The cross-border malaria information system (Saldanha et al., submitted) was also approved by the CNIL ( $\left.{ }^{\circ} 2135463\right)$.

\section{Results}

\section{Epidemiological description of malaria cases within the French Guiana border area (STG health center):}

During the study period from January 1, 2017 to January 31, 2018, 219 people were infected with $P$. vivax (primary attack). The median age was 22 years [interquartile range (IQR) $=19.9-24.27$ ]; $50.7 \%$ $(n=111 / 219)$ of cases were less than 18 years old. The sex ratio was $1.23(n=121 / 98)$. A significant portion $(27 \%, \mathrm{n}=59)$ experienced at least one relapse of $P$. vivax infection, $6.9 \%(\mathrm{n}=15)$ experienced 2 relapses and $1 \%(n=3)$ presented 3 relapses. The spatial and temporal distribution of cases suggest that since May 2017 the risk of infection steadily increased among the residents of STG, with a peak in November 2017. The attack rate was 5.5\% in the STG municipality, reaching $51.4 \%$ in the TroisPalétuviers neighborhood (Figure 2). Children ( $<18$ years old) had a significantly higher risk of relapse $(p<0.005$, OR 4.03 [2.00-8.31]). The risk of relapse was the same between males and females. Residents of the Trois-Palétuviers neighborhood also had a higher risk of relapse compared to other STG neighborhoods $(p=0.037)$.

Although $16.5 \%(n=31 / 188)$ of patients had a G6PD activity below the laboratory reference values ( $N$ : $10-$ $14 \mathrm{U} / \mathrm{HgL}$ ), only $6.9 \%(\mathrm{n}=13 / 188)$ of patients had an intermediate G6PD deficiency and no severe deficiency was reported (Supplement S1). In January 2018, 68.5\% ( $n=150)$ of malaria cases received complete treatment (chloroquine and primaquine), $6.4 \%(n=14)$ were only treated with chloroquine due to a contraindicated primaquine treatment (pregnancy, breastfeeding or G6PD deficiency), $1.3 \%(n=3)$ were treated in another health center and $23.7 \%(n=52)$ were lost in follow-up and treated only by chloroquine.

\section{Entomological findings}

From August to November 2017, 1,246 females of four Anopheles species were collected, and $97.4 \%$ of them were captured with the Mosquito-Magnets. Anopheles darlingi was the predominant Anopheles 
species ( $n=1,230$ ), representing $99.2 \%$ of captured anophelines (excluding seven Anopheles unidentified at the species level). Other species collected were Anopheles intermedius (eight individuals in August), An. nuneztovari (one in September) and An. oswaldoi (one in October).

Of the 1,218 Anopheles tested for malaria by PCR, two female An. darlingi were found positive for $P v$. One was captured on August $3^{\text {rd }}$ (between 5:30 and 8:00am) and one on September $13^{\text {th }}$ (between 20:00 and 21:00pm). Both infected mosquitoes were captured in Mosquito-Magnets placed on the outskirts of the neighborhood, less than 50 meters from the forest. Prevalence was estimated at $0.19 \%$ in August $(n=514)$ and $0.17 \%$ in September $(n=590)$.

The density of An. darlingi at the village outskirts was higher in August and September than in October and November $2017(p=0.045)$, with no significant peak in August (Figure 3). Anopheles darlingi was captured at all hours of the collection period. It was also the only Anopheline species found biting inside households in the evening in August.

\section{Seasonality and comparison of the border area between French Guiana and Brazil, 2007-2017}

Malaria infection demonstrated a distinct seasonal variation on the French Guiana and Brazilian border with peaks in November (Figure 4). Thus, most malaria cases in French Guiana (63.1\%, n=3346/5385) occurred during the dry season (approximately from July to December). The 2017 outbreak clearly demonstrated the same profile as previous epidemic profiles. In recent years, malaria infections are mostly due to $\mathrm{Pv}$ on both sides of the border.

\section{Epidemic profile analysis}

The total number of cases reported in registries during this period (from January 1, 2017 to January 31, 2018) was 1,664 (1,434 in Brazil, 182 in French Guiana). Forty eight (48) cases were associated with unspecified localities of residence and were ignored (nine were notified in Brazil, with Brazil as the country of residence; the 39 remaining cases were notified in French Guiana without any mention of country of residence). Figure 5 illustrates the results of the epidemic curve clustering. A dendrogram was dissected to define four clusters that corresponded to both low intra-cluster and high inter-cluster variances. Clusters were ranged chronologically by considering the time interval during which the weekly case numbers reached their maximum, corresponding to the inflection points of the cumulated curves. The inflection point corresponded approximately to the moment when the number of cases reached $50 \%$ of the total number of the cases during the whole considered period (Figure 5). All localities of the first two clusters are Brazilian (Figure 6 and Supplement S2). Figure 6 is a map of the localities and clusters. All localities are situated in the northern part of the study area. Amerindian areas were reached first, demonstrating high incidence rates compared to the French STG or Brazilian Oiapoque city centers (Figure 6). Cluster 1 included two Amerindian localities, with a much earlier start of the epidemic than elsewhere (between weeks 21-25) (Figure 6 and Supplement S2). The second cluster included two other Amerindian villages (mostly Palikur inhabitants) close to the first cluster (Figure 6 and Supplement S2). Cluster 3 gathered French Guianese neighborhoods and the Oiapoque city center, including Trois- 
Palétuviers and Brazilian villages which broadened the progressive spread of the outbreak (Figure 6 and Supplement S2). Finally, cluster 4 represented STG and Oiapoque city centers and a continual expansion of the outbreak, in particular in the south-eastern Kumarumã (Galibi-marworno) village (Figure 6 and Supplement S2).

\section{Outbreak control measures}

In French Guiana, the implemented control measures included the creation of a dedicated medical malaria team with an infectious disease physician, a Cayenne hospital nurse and cultural mediators from a local NGO (DAAC). This team was implemented in November 2017and financed by the regional health agency of French Guiana and Cayenne hospital. It has contributed to improving diagnosis and follow-up of malaria cases in STG. This medical team used cars or canoes to directly visit households with malaria cases in order to expediate treatment and control transmission. The medical intervention implemented malaria testing and G6PD blood testing and provided complete malaria treatment (chloroquine and primaquine). In order to facilitate outbreak control, the French National Agency for Medicines and Health Products Safety (ANSM) implemented a temporary accelerated treatment authorization procedure in order to quicken the access to primaquine. Vector control measures were also deployed to reduce contact between people and Anopheles mosquitoes: long-lasting insecticidal nets (LLINs) were distributed to every malaria positive case, their family members and also to the entire population of Trois-Palétuviers. Indoor residual sprayings with deltamethrin were also realized. Good practices to avoid mosquito bites were taught orally to individuals and to the communities in public spaces.

In Brazil, active case detection by thick and thin smears along with indoor residual sprayings were performed in Oiapoque city, as well as in the Taparabú village (a Brazilian village facing the TroisPalétuviers French Guianese neighborhood).

Since the earliest phase of the epidemic, transborder communication has been established between the municipality of Oiapoque and the regional health agency of French Guiana in an aim to keep both parties informed of local trends and to sustain preventive measures.

\section{Discussion}

The 2017 outbreak peak of malaria cases presented a greater intensity with a similar pattern compared to previous seasonal peaks in both the French Guiana and Brazilian border areas. Several factors may explain this renewed increase of malaria infections. Firstly, there has been a shift from $P$. falciparum to $P$. vivax infections in this area over the last decade with greater difficulty (contraindication, availability of G6PD blood test and treatment) in treating with primaquine and thus in avoiding relapses [6, 17]. Secondly, hikes in infections may be due to environmental causes such as the exceptional rainy season in 2017 - which can raise vector density - and the impacts of increased deforestation in Amazonia [18, 19]. Thirdly, in 2017, a new municipal government was inaugurated in Oiapoque. With that, leadership roles shifted in the Special Indigenous Health District (DSEI), an entity responsible for providing health care to indigenous people in Oiapoque. At the onset of this type of change in power dynamics, there is 
often an administrative discontinuity of local governments, which may have consequences on public services [20]. Finally, political and economic regional crises, particularly in Venezuela but also in Brazil, have led to large-scale human migration into Brazil and is associated with an increase of illegal gold mining $[4,21]$. Unexpectedly, our results on incidence and spatial distribution revealed an additional human factor within the specific population of Amerindians. The study of the epidemic dynamics tends to show that the number of cases began increasing in Brazilian Amerindian villages before increasing on the French side of the border. These communities appeared to be particularly at risk for malaria infection and participated in factors driving this epidemic. Remote Amerindian communities have specific behaviors, precarious living conditions, poor access to health care, higher mobility, as well as commercial, cultural or family links among populations from both sides of the border. This may explain the crossborder epidemic dynamic, and these communities represent a key population for malaria control. However, further investigations should be conducted to: i) clearly identify these hypothetical mechanisms; ii) confirm that such a scenario may have occurred in previous years; iii) show that observed events did not occur independently. Indeed, studies of the transmission dynamics for each Plasmodium spp. genotype could help provide an overview of the spread of infection in this cross-border population.

Present and past studies show the influence of vector dynamics on malaria transmission [12, 22]. In 2017, the heaviest rainfall hit the area since 2000 and may have had an impact on mosquito density [18]. Our collections of An. darlingi confirm the well-known exo/endophagic behavior of this vector, and the need to prevent host-vector contact inside households (with mosquito nets) and outside (use of repellants and loose clothing). Several recent publications refer to insecticide resistance within this species in Brazil [23], and also increased daytime activity [22]. Thus, these factors contribute to the challenge of vector control in addition to the difficulty of indoor insecticide spraying and providing long-lasting insecticidal nets for remote and mobile communities.

Because of the presence and profusion of infected females in August and September - before the human peak in November -, An. darlingi was likely the main Anopheline species involved in the emergence and dynamics of this outbreak. On the French Guiana side, most malaria cases occurred in neighborhoods with dense vegetation (Blondin and Trois-Palétuviers) where high densities of An. darlingi were reported both in the past and present [12,24]. Blondin and Trois-Palétuviers are also the farthest from the health center, with $15 \mathrm{~min}$. and $45 \mathrm{~min}$. of travel time by canoe, respectively. This isolation may participate in foregoing medical treatment and may explain the higher rate of relapse, particularly in the Amerindian (mainly Palikur) neighborhood of Trois-Palétuviers. In considering the high attack rate in Trois-Palétuviers, our results suggest that the combination of vector density, exchanges and mobility among Brazilian Amerindian Palikur villages, isolation and high relapse rates helps explain the intensity of the 2017 outbreak on the French Guiana side of the border.

Outbreak control measures in French Guiana enabled the creation of a specific malaria team with community health mediators from November to February. This team administered radical treatment to a large majority of cases. A previous retrospective study in the same area did not deploy the same strategy 
and reported less than $5 \%$ complete treatments [17]. This highlights the importance of optimizing responses by integrating community mediators.

This area presented the same epidemiological setting on both sides of the border, yet possesses two distinct programs for diagnosis and treatment, complicating the implementation of public health programs. However, health services on both sides of the border area have favorable workforces and technological conditions to positively contribute to successful malaria control during their routine activities. Indeed, Brazilian and French Guianese malaria cases appeared to be related in space (border area) and time, but also experienced by the same Amerindians communities, notably the Palikur people. The Palikur are a mobile Amerindian community and they commonly go from one side of the border to the other, particularly to and from the Brazilian villages of Kouméné and Amomi. These two villages are considered the spiritual center for the Palikur communities living in Trois-Palétuviers, Philogène or in their Blondin French Guianese neighborhoods [25]. Amerindian health centers are autonomous in Brazil and care is given by and for Amerindians through the National Indian Foundation (FUNAI). However, improvements may be needed in collaborative efforts between Brazilian health authorities and the FUNAI, cross-border alliances and community mobilization to achieve malaria control in both countries.

\section{Conclusion}

The current findings demonstrate a potential increase in malaria cases in an area with otherwise declining numbers. This is a transborder area where human mobility and remote populations challenge malaria control programs. P. vivax elimination requires greater, well-coordinated efforts and bordertranscending malaria control. New strategies may be vital to achieving elimination, particularly in Amerindians communities.

\section{Declarations}

\section{Ethics approval and consent to participate}

The French Guianese database was anonymized and declared to the Commission Nationale Informatique et Libertés (CNIL) (authorization №1939018). Brazil's surveillance registries database was anonymized prior to being sent. The cross-border malaria information system (Saldanha et al., submitted) was also approved by the CNIL (N²135463).

\section{Consent for publication}

Not applicable

\section{Availability of data and materials}

The datasets generated and analyzed during the current study are not publicly available due to the requirement of special authorization to transfer databases provided by the CNIL. Upon prior CNIL 
authorization, the datasets can be made available from the corresponding author upon reasonable request.

\section{Competing interests}

The authors declare that there is no conflict of interest.

\section{Funding}

This study was funded by the French Guianese Regional Health Agency and by the European Funds for Regional Development, "ELIMALAR" NSynergie: GY0012082 and "CONTROLE” N'Synergie: GY0010695. It also benefited from: Project "Fighting Malaria: From Global 'War' to 'Local Guerillas' at International Borders", funded by the Grand Challenges Explorations (GCE) Round 18 program of the Bill and Melinda Gates Foundation (OPP1171795); the project "GAPAM-Sentinela", funded by the Guyamazon program (IRD, CIRAD, French Guiana regional authorities, the French Embassy in Brazil, FAPEAP, FAPEAM); the Joint International Laboratory “Cross-border climate, environment and vector-borne diseases observatories - Sentinel site of the Brazilian climate and health observatory" (LMI Sentinela) gathering IRD, FIOCRUZ, Brasilia University (UnB) and partners. The funding bodies had no role in the study and in the publication process.

\section{Authors' contributions}

$E M, E R, G L$ analyzed the data. EM, ID, ER conceptualized the study. EM, ID, GL, AG, MSG, AS, YE, JR, DD, $L M, D M, F D, M D, V A, M N, R S$, ER contributed regional microbiological, entomological and epidemiological data. EM and ER wrote the first draft with the inputs from all authors. The manuscript has been approved in the final version by all the coauthors.

\section{Acknowledgements}

The authors gratefully acknowledge the DAAC association and their cultural mediators, Collectivité Térritoriale de la Guyane, Olivier Moriceau, Stephane Gauduchon, Dr Philippe Travers, Dr Bastien Bidaud, Dr Paul Brousse, Dr Nicolas Garçeran, Dr Mélanie Gaillet, Dr Céline Michaud and the medical Brazilian and French Guianese team for their work to control this outbreak. We would like to thank Jean Issaly, Romuald Carinci and Pascal Gaborit for their entomological fieldwork. Finally, we would also like to thank The Secretaria de Saùde, especially the Vigilância Ambiental team of the Oiapoque municipality, Ms Mara Midena and the Programa Nacional de Controle e Prevenção da Malaria of the BInteressant?o quicken the access to primaquine.ed authorization proceduren: overall necessityu f FGrégionale'razilian Ministry of health.

\section{References}

1. WHO. World malaria report, 2017. Geneva: World Health Organization; 2017. http://www.who.int/malaria/publications/world_malaria_report/en/ 
2. Ferreira MU, Castro MC. Challenges for malaria elimination in Brazil. Malar J. 2016 20;15(1):284.

3. Merle N. Plan de lutte contre le paludisme en Guyane. ARS Guyane; 2015. https://www.guyane.ars.sante.fr/system/files/2017-06/palu\%20plan\%202015-2018.pdf

4. Douine M, Musset L, Corlin F, Pelleau S, Pasquier J, Mutricy L, et al. Prevalence of Plasmodium spp. in illegal gold miners in French Guiana in 2015: a hidden but critical malaria reservoir. Malar J. 2016 Jun 9;15:315.

5. Mosnier E, Roux E, Cropet C, Lazrek Y, Moriceau O, Gaillet M, et al. Prevalence of Plasmodium spp. in the Amazonian Border Context (French Guiana-Brazil): Associated Factors and Spatial Distribution. Am J Trop Med Hyg. 2020;102:130-41.

6. Musset L, Pelleau S, Girod R, Ardillon V, Carvalho L, Dusfour I, et al. Malaria on the Guiana Shield: a review of the situation in French Guiana. Mem Inst Oswaldo Cruz. 2014 Aug 13;0:0.

7. Ardillon V. Surveillance du paludisme. Bulletin périodique: novembre 2017 à janvier 2019. Le Point Epid. 2018 Feb 1.

8. Bousema T, Griffin JT, Sauerwein RW, Smith DL, Churcher TS, Takken W, et al. Hitting hotspots: spatial targeting of malaria for control and elimination. PLoS Med. 2012 Jan;9(1):e1001165.

9. Howes RE, Battle KE, Mendis KN, Smith DL, Cibulskis RE, Baird JK, et al. Global Epidemiology of Plasmodium vivax. Am J Trop Med Hyg. 2016 Dec 28;95(6 Suppl):15-34.

10. Wangdi K, Gatton ML, Kelly GC, Clements ACA. Cross-border malaria: a major obstacle for malaria elimination. Adv Parasitol. 2015 Jun;89:79-107.

11. Epelboin Y, Chaney SC, Guidez A, Habchi-Hanriot N, Talaga S, Wang L, et al. Successes and failures of sixty years of vector control in French Guiana: what is the next step? Mem Inst Oswaldo Cruz [Internet]. 2018 Mar 12 [cited 2018 Jun 28];113(5). Available from:

https://www.ncbi.nlm.nih.gov/pmc/articles/PMC5851058/

12. Adde A, Roux E, Mangeas M, Dessay N, Nacher M, Dusfour I, et al. Dynamical Mapping of Anopheles darlingi Densities in a Residual Malaria Transmission Area of French Guiana by Using Remote Sensing and Meteorological Data. PloS One. 2016;11(10):e0164685.

13. WHO. Dépistage du déficit en G6PD pour une utilisation sans risque de la primaquine dans le traitement radical du paludisme à P. vivax ou P. ovale: note d'orientation. Geneva: World Health Organization; 2017. https://apps.who.int/iris/bitstream/handle/10665/258603/WHO-HTM-GMP2016.9-fre.pdf;jsessionid=2449ACD4ED77309363A1F5E6EC78BD9B? sequence=1

14. Hanf M, Stéphani A, Basurko C, Nacher M, Carme B. Determination of the Plasmodium vivax relapse pattern in Camopi, French Guiana. Malar J. 2009 Dec 4;8:278.

15. Vezenegho SB, Adde A, Gaborit P, Carinci R, Issaly J, Pommier de Santi V, et al. Mosquito magnet ${ }^{\circledR}$ liberty plus trap baited with octenol confirmed best candidate for Anopheles surveillance and proved promising in predicting risk of malaria transmission in French Guiana. Malar J. 2014 Sep 26;13:384.

16. Snounou G, Viriyakosol S, Zhu XP, Jarra W, Pinheiro L, do Rosario VE, et al. High sensitivity of detection of human malaria parasites by the use of nested polymerase chain reaction. Mol Biochem Parasitol. 1993 Oct;61(2):315-20. 
17. Nacher M, Stefani A, Basurko C, Lemonnier D, Djossou F, Demar M, et al. The burden of Plasmodium vivax relapses in an Amerindian village in French Guiana. Malar J. 2013 Oct 24;12:367.

18. Météo France. Bulletin climatique annuel 2017. Météo France; 2017. http://www.meteofrance.gp/climat/pluies-annuelles/rr_an_guyane

19. Chaves LSM, Conn JE, López RVM, Sallum MAM. Abundance of impacted forest patches less than 5 km2 is a key driver of the incidence of malaria in Amazonian Brazil. Sci Rep. 2018 May 4;8(1):7077.

20. Nogueira $F$ do A. Continuidade e descontinuidade administrativa em governos locais: fatores que sustentam a ação pública ao longo dos anos. 2006 May 8. http://bibliotecadigital.fgv.br/dspace/handle/10438/2423

21. Grillet ME, Villegas L, Oletta JF, Tami A, Conn JE. Malaria in Venezuela requires response. Science. 2018 02;359(6375):528.

22. Vezenegho SB, Adde A, Pommier de Santi V, Issaly J, Carinci R, Gaborit P, et al. High malaria transmission in a forested malaria focus in French Guiana: How can exophagic Anopheles darlingi thwart vector control and prevention measures? Mem Inst Oswaldo Cruz. 2016 Sep;111(9):561-9.

23. Galardo AKR, Póvoa MM, Sucupira IMC, Galardo CD, Santos RLCD. Anopheles darlingi and Anopheles marajoara (Diptera: Culicidae) susceptibility to pyrethroids in an endemic area of the Brazilian Amazon. Rev Soc Bras Med Trop. 2015 Dec;48(6):765-9.

24. Adde A, Dusfour I, Vezenegho SB, Carinci R, Issaly J, Gaborit P, et al. Spatial and Seasonal Dynamics of Anopheles Mosquitoes in Saint-Georges de l'Oyapock, French Guiana: Influence of Environmental Factors. J Med Entomol. 2017 May 1;54(3):597-605.

25. Passes A. Both Omphalos and Margin: On how the Pa'ikwené (Palikur) see themselves to be at the center and on the edge at the same time. Comp Arawakan Hist Rethink Lang Fam Cult Area Amazon. 2002;171-195.

\section{Supplementary Data}

Supplement S1: Distribution of G6PD deficiency according to the WHO definition of French Guianese participants

Supplement S2: Results of clusters for selected localities in the cross-border area between French Guiana and Brazil, January 2017-January 2018. FUNAI: Fundação Nacional do Índio, Brazil; SIVEP-Malária:

Sistema de Vigilância Epidemiológica da Malária, Brazil; CDPS: Delocalized Centers for Prevention and Care, Cayenne Hospital, French Guiana; BR: Brazil; GF: French Guiana

Supplement S3: Graphical abstract of the main result of the malaria outbreak investigation, border between French Guiana and Brazil, 2017

\section{Figures}




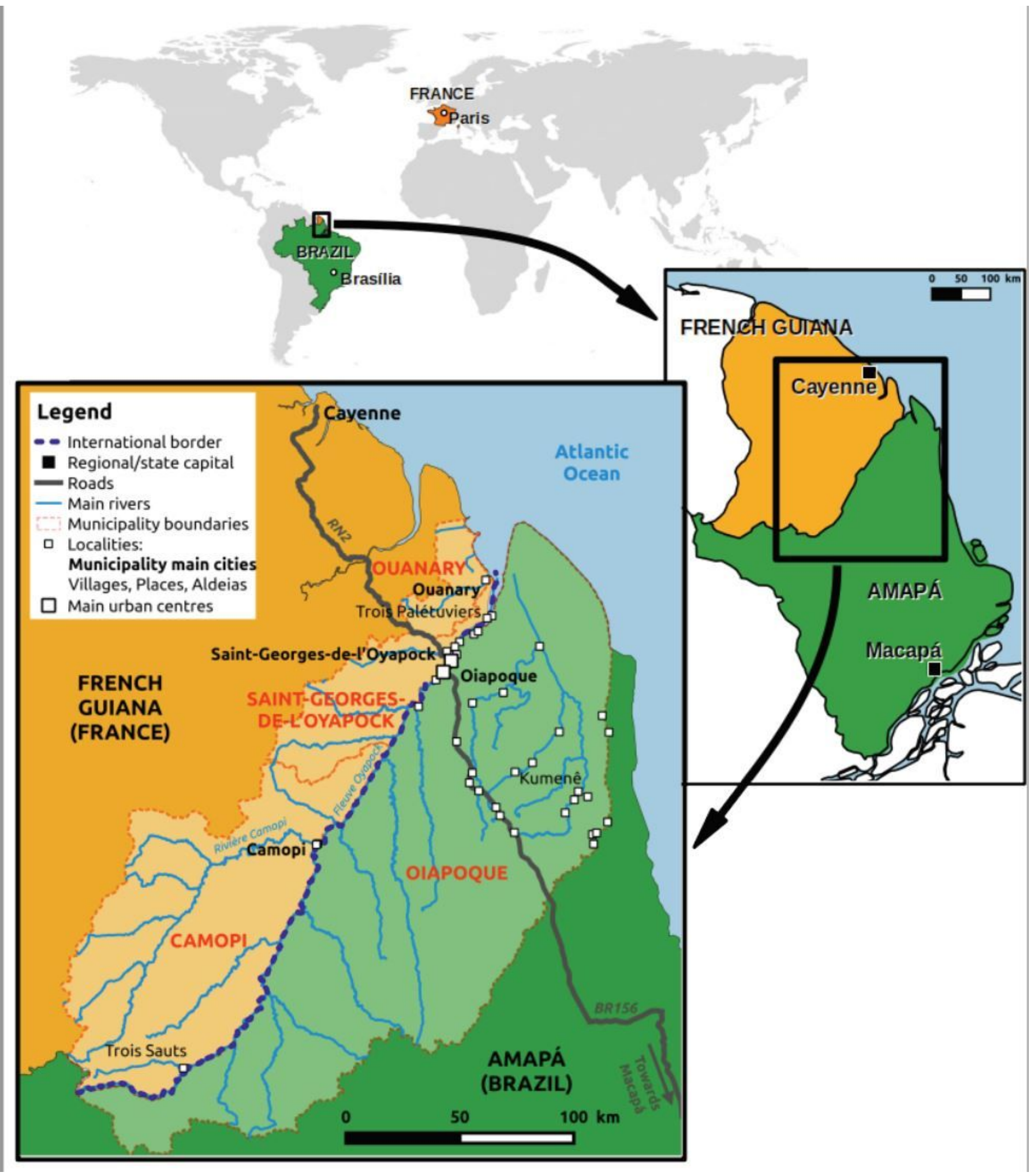

Figure 1

Map of the border area between Brazil and French Guiana 


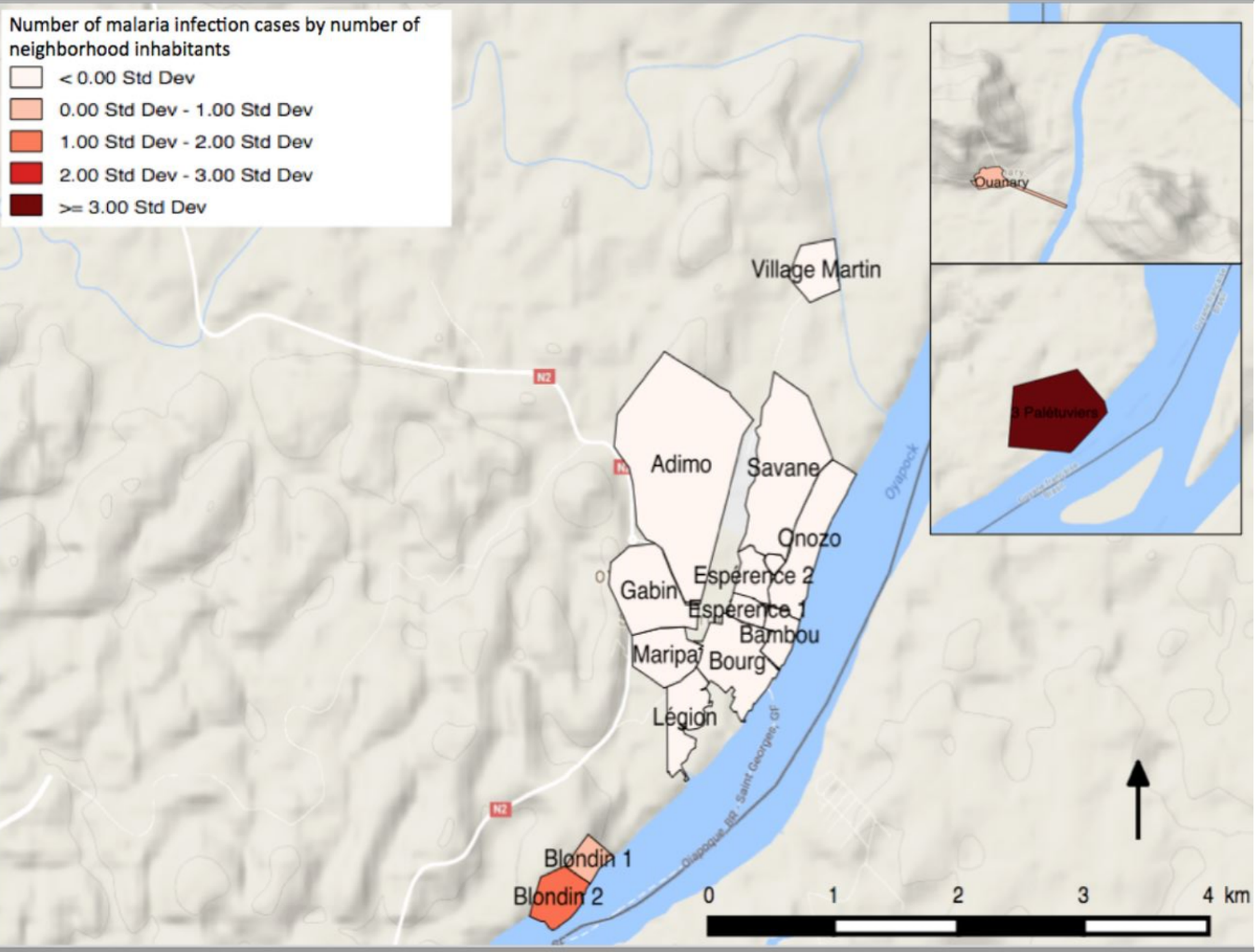

Figure 2

Mapping of the incidence of P. vivax infection cases of neighborhood inhabitants of the French Guiana border area (Saint Georges de l'Oyapock and Ouanary villages), January 2017-January 2018. 


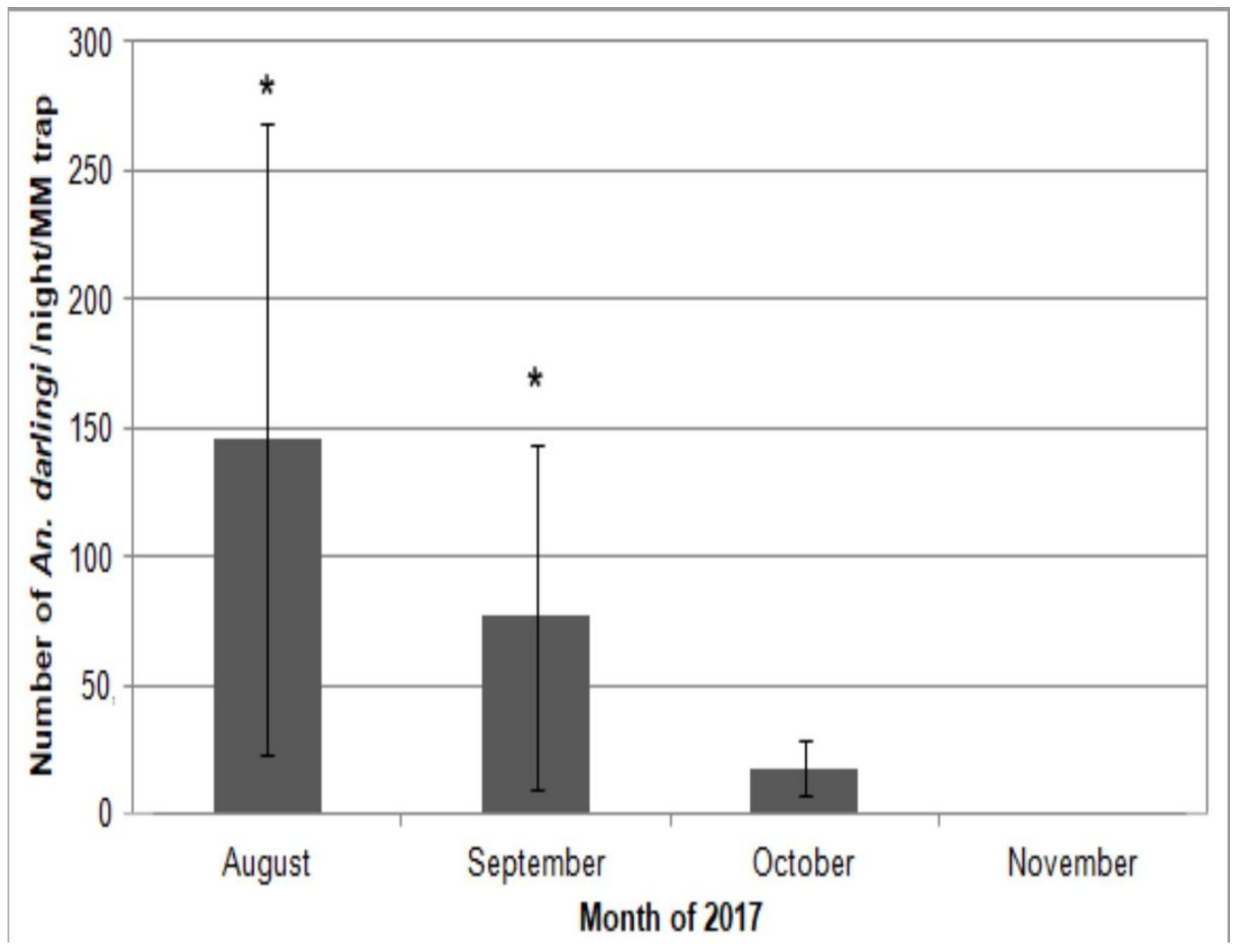

Figure 3

Monthly mean number of Anopheles darlingi captured by night, Trois-Palétuviers, French Guiana, August to November 2017. The asterisks $\left(^{*}\right)$ represent the months during which one mosquito infected with Plasmodium vivax was captured. Only the collections of Mosquito-Magnets at the village outskirts were considered. 
French Guiana (border municipalities)

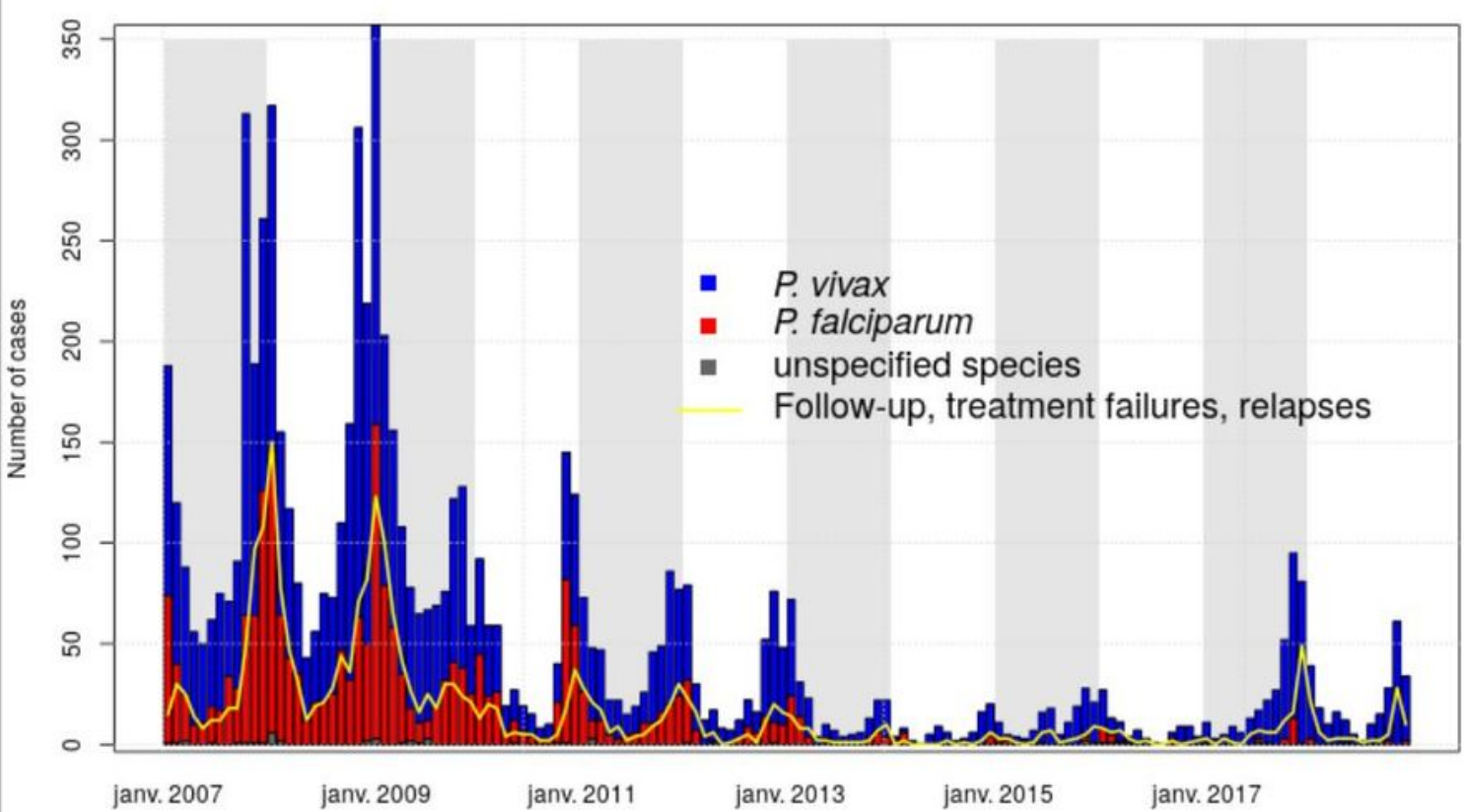

Brazil (Oiapoque municipality)

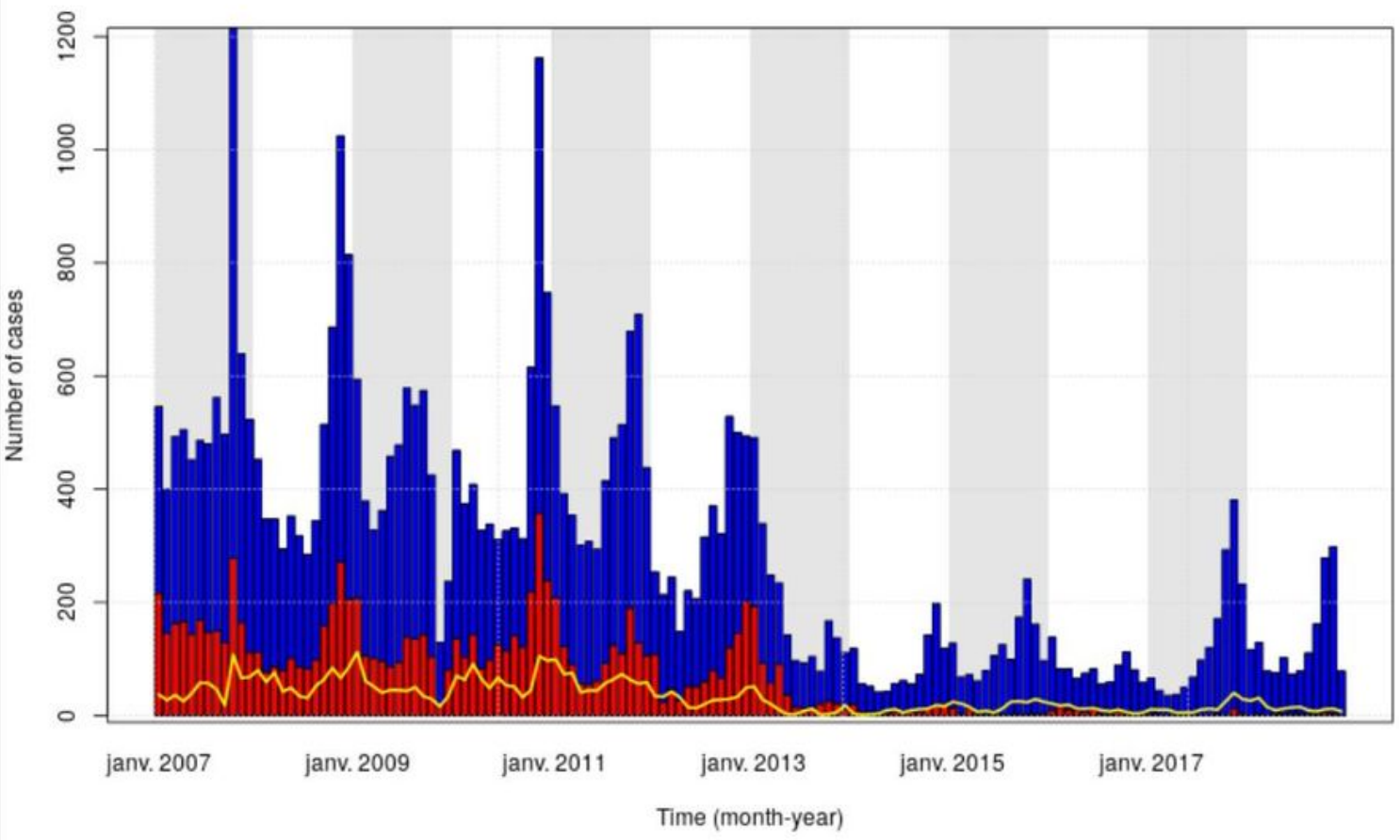

Figure 4

Monthly temporal dynamics of malaria cases in French Guiana and Brazil border areas. 


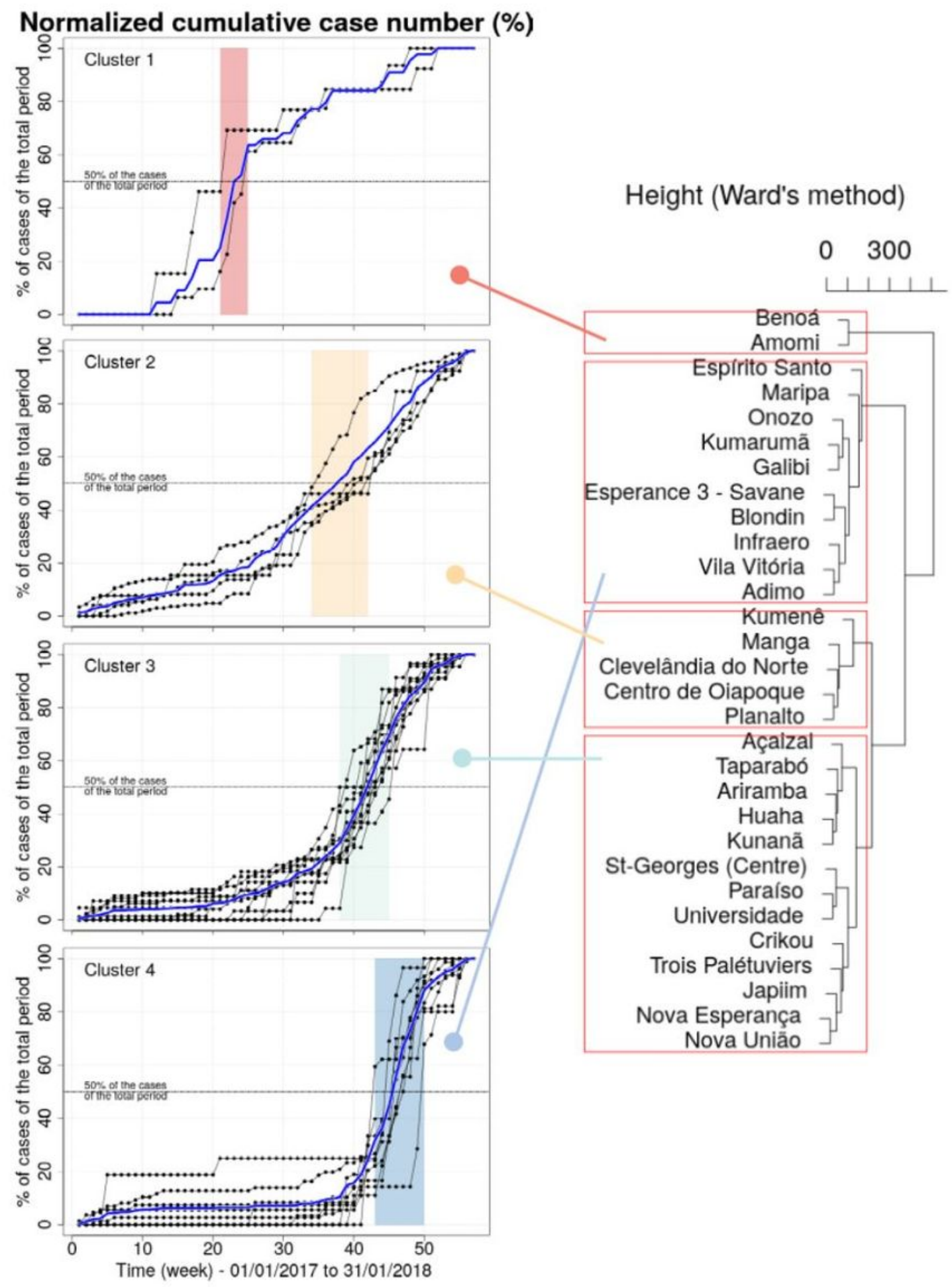

\section{Figure 5}

On the right: a dendrogram resulting from hierarchical clustering (Euclidean distance and Ward aggregation method). On the left, the normalized cumulated case numbers according to the clusters on the cross-border area between French Guiana and Brazil, January 2017-January 2018. The period during which $50 \%$ of the total case number is reached, for all the locality clusters, is represented in color. The thick blue curve represents the normalized cumulated curve of the total number of cases per cluster. 


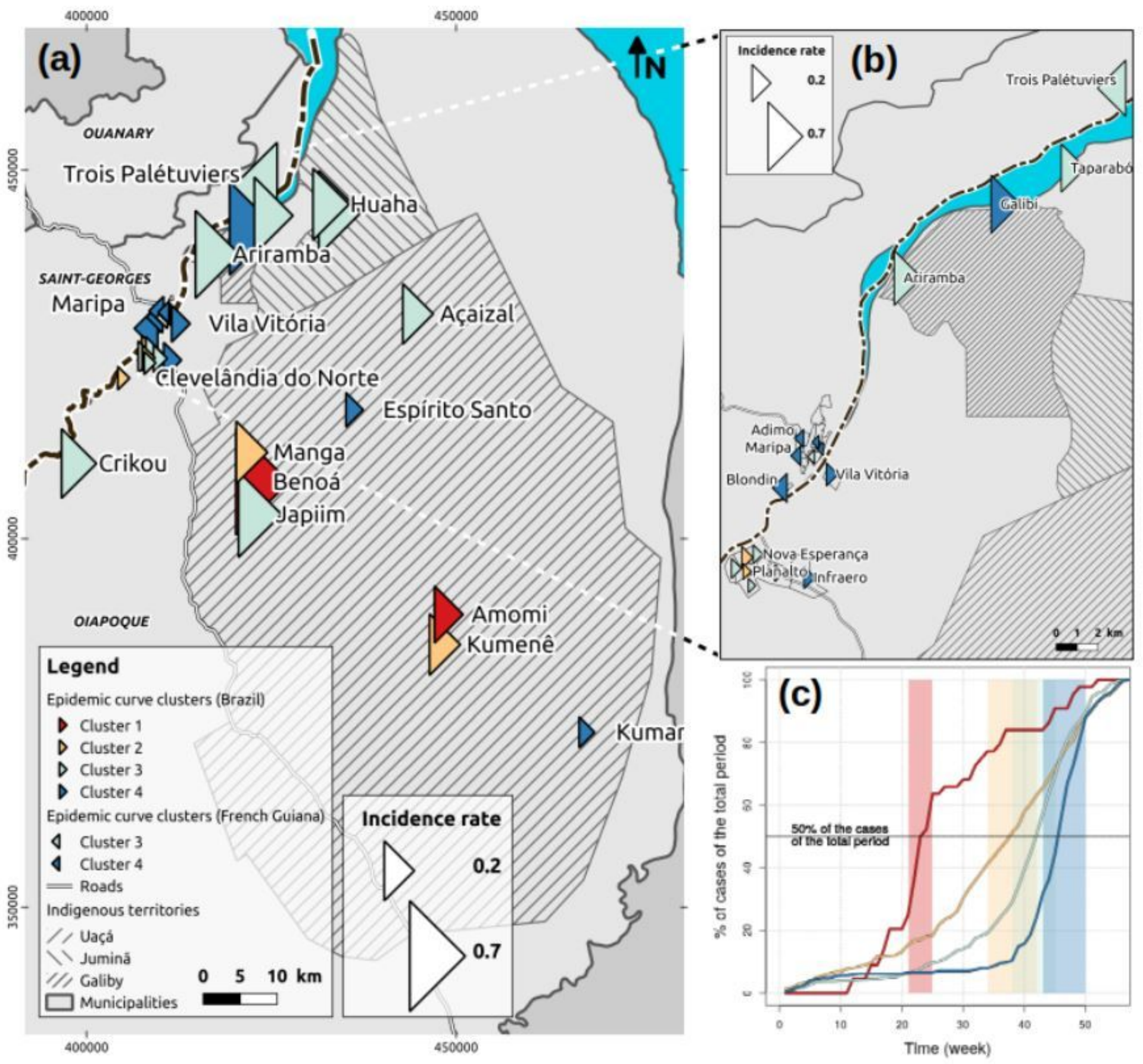

Figure 6

Cartographic representation of the localities and clusters on the cross-border area between French Guiana and Brazil, January 2017-January 2018: a) All cross-border localities included in the cluster analysis; b) focus on the border; $c$ ) normalized cumulated curves for the averaged number of cases per week and per cluster (same color code as in Figure 1). The size of the triangles is proportional to the incidence rate. The colors of the triangles correspond to the color code used in Figure 1. Bottom right: normalized cumulated curves for the averaged number of cases per cluster (same color code as in Figure 1).

\section{Supplementary Files}


This is a list of supplementary files associated with this preprint. Click to download.

- SupplementarydataTable1and2Outbreakmalariacopie.docx

- graphicalabstractoutbreak.pdf 